\title{
PROPERTIES OF THE PHASE TRANSITION IN THE TRANSVERSE ISING MODEL
}

\author{
L. De Cesare $^{\dagger}$, I. Rabuffo ${ }^{\dagger}$, D. I. Uzunov ${ }^{\dagger}$ \\ †Dipartimento di Scienze Fisiche "E. R. Caianiello", Università di Salerno, I-84081 Baronissi, Salerno, Italy, and Istituto \\ Nazionalle per la Fisica della Materia, Unità di Salerno, Italy \\ ${ }^{\ddagger}$ CPCM Laboratory, G. Nadjakov Institute of Solid State Physics, Bulgarian Academy of Sciences, BG-1784 Sofia, Bulgaria
} (Received April 24, 1998)

\begin{abstract}
The phase transition properties of the quantum spin-1/2 Ising model in a transverse magnetic field are reconsidered with the help of the mean field approximation, the Ginzburg criterion, and the renormalization group theory. A special attention is paid to the phase transitions whose properties are determined by variations of the external transverse field and to the low-temperature phase transitions due to temperature variations. The classical-to-quantum crossover is investigated in details. A new crossover effect is established and discussed.
\end{abstract}

Key words: equation of state, critical exponents, phase transition, renormalization group, quantum critical phenomena.

PACS number(s): 05.70.Jk, 64.60.Fr, 64.60.Kw, 75.40.Cx

\section{INTRODUCTION}

In this report, we shall consider the classical-toquantum dimensional crossover (CQC) $[1,2]$ in systems described by the quantum spin-1/2 Ising model in a transverse magnetic field which is often referred to as the "transverse Ising model" (TIM). The problem for the CQC in quantum systems has been recently raised again in view of the current interest in the properties of low-dimensional magnetic systems. In the last years the classical results $[1,2]$ have been reconsidered and new developments of the theory have been achieved [3]. Using the preceding mean-field (MF) [4,5] and renormalization group (RG) [1,2] results here we shall investigate the CQC within TIM in details. Besides, several new results for the critical properties of TIM obtained by means of the MF, the Ginzburg criterion (GC) [2] and the RG of TIM, are reported. We shall show that the present MF investigation can be used to introduce another crossover, namely, the crossover from the high-temperature (HT) to low-temperature (LT) critical properties of TIM; hereafter this crossover is referred to as HLTC.

The TIM is given by the Hamiltonian

$$
\mathcal{H}=-\frac{1}{2} \sum_{i j} S_{i}^{z} S_{j}^{z}-\Gamma \sum_{i} S_{i}^{x},
$$

where $S_{i}, i=(x, y, z)$ are the components of the spins taken to have a magnitude $S=1$ (instead of the original $S=1 / 2), J_{i j}$ is the exchange interaction and $\Gamma$ is the magnitude of the transverse field. We shall assume that TIM is defined on a $D$-dimensional regular lattice with the lattice spacing $a$ and the volume $V=\left(L_{1} \ldots L_{D}\right)$, where the $L_{i}$ are large ("infinite") dimensions: $L_{i} \gg a$; $i=(1, \ldots, D)$. The applications of this model to real magnetic and ferroelectric systems have been thoroughly enumerated and discussed in Ref. [4]. Previous theoretical work on TIM has been largely based on the MF [5], series expansions [6], cumulant expansions [4], and RG $[7,8]$.

The main conclusion of the preceding investigations $[1,2]$ is that the asymptotic critical behaviour of the $D$ dimensional TIM undergoes a simple dimensional CQC of the type $D \rightarrow(D+1)$ when the critical temperature $T_{c}$ becomes equal to zero. The critical behaviour of TIM at $T_{c} \neq 0$ belongs to the universality class $(n, D)$ with $n=1 ; n$ is the number of the order parameter components [2]. This is the usual ("classical") critical behaviour in a close vicinity of the "finite" critical point $\left(T_{c} \neq 0\right)$, where the quantum correlations in the system are irrelevant. Within the MF approximation (Section III) the classification of the phase transitions in universality classes becomes senseless because all standard second-order transitions belong to one and the same universality class - the MF description, or the "MF universality class". We shall call the critical behaviour in this case a "classical MF behaviour".

When $T_{c} \rightarrow 0$ the quantum correlations in TIM become relevant to the critical behaviour because the thermal wave length $\lambda_{T} \sim(1 / T)[2]$ tends to infinity. These quantum correlations are the main reason for the appearance of $\mathrm{CQC}[1,2]$.

Within the framework of the MF approximation we shall show that several HT and LT phase transitions in TIM exhibit nonuniversal features. The GC will be used to determine the domains of validity of the MF results in the $(T, \Gamma)$ phase diagram. Besides, a quantum version of the $\mathrm{GC}$ will be derived to demonstrate the $\mathrm{CQC}$ at extremely low temperatures.

The RG recursion method of Wilson and Fisher combined with a generalized $\epsilon$-expansion will be applied to show that only the true ("quantum") zero-temperature critical behaviour exhibits a CQC. The onset of CQC can be observed at extremely LT evaluated in this paper. We demonstrate that the quantum effects are irrelevant for the bigger part of the HT and LT phase transitions in TIM. On the basis of this circumstance and owing to 
the substantial difference between the HT and LT phase transition properties, we introduce the HLTC.

\section{FIELD COUNTERPART OF THE TRANSVERSE ISING MODEL}

The results in preceding investigations [4,8] can be used to show that the field counterpart of the TIM can be written in the form of a $\phi^{4}$-Hamiltonian of the Bose field $\phi(q)$, where the $(D+1)$-dimensional vector $q=\left(\omega_{l}, \mathbf{k}\right)$ is given by the Matsubara frequency $\omega_{l}=2 \pi l T$ (for $\hbar=1)$ and the wave vector $\mathbf{k}=\left\{k_{i} ; i=1, \ldots, D\right\}$. The $\phi^{4}$-Hamiltonian $\left(\mathcal{H}=H / T, k_{B}=1\right)$ is

$$
\begin{aligned}
\mathcal{H} & =\frac{1}{2} \sum_{q}\left[\nu_{l}^{2}+1-\frac{J(k)}{\Gamma} \operatorname{th}(\beta \Gamma)\right]|\phi(q)|^{2} \\
& +\frac{1}{V} \sum_{q_{i}} \mathcal{U}\left(\Gamma, J ; q_{i}\right) \phi\left(q_{1}\right) \phi\left(q_{2}\right) \phi\left(q_{3}\right) \phi\left(-q_{1}-q_{2}-q_{3}\right),
\end{aligned}
$$

where $\nu_{l}=\omega_{l} / 2 \Gamma, V$ is the volume, and the bare vertex $\mathcal{U}$ is an analytical function of $q$. For our purposes the $q$-dependence of $\mathcal{U}$ is irrelevant and we shall present the value of this quantity for $q=0$, namely, $u=\mathcal{U}\left(\Gamma, J ; q_{i}=\right.$ $0)$, given by

$$
u=\frac{a^{D} J^{2}(0)}{4 ! T^{2}} \bar{u}
$$

where

$$
\bar{u}=3\left(\frac{T}{\Gamma}\right)^{3}\left[\operatorname{th}\left(\frac{\Gamma}{T}\right)-\frac{\Gamma}{T}+\frac{\Gamma}{T} \operatorname{th}^{2}\left(\frac{\Gamma}{T}\right)\right] .
$$

As is usual, we apply the long wavelength approximation (LWLA) [9] to the quadratic $\left(\phi^{2}-\right)$ part of the model (2). We shall use the example of the $n n$ interactions in a simple cubic lattice, but this has no consequences for the results in this report. Performing standard calculations in the LWLA and some transformations of the wave components $k_{i}$ we obtain the quadratic part $\mathcal{H}_{2}$ of the Hamiltonian (2) in the form

$$
\mathcal{H}_{2}=\frac{1}{2} \sum_{q} G_{0}^{-1}(q)|\phi(q)|^{2}
$$

The (bare) correlation function will be

$$
G_{0}^{-1}(q)=\left(t_{0}+k^{2}+\nu_{l}^{2}\right)
$$

with

$$
t_{0}=\left[1-\frac{J}{\Gamma} \operatorname{th}\left(\frac{\Gamma}{T}\right)\right]
$$

where $J \equiv J(0)=2 D J_{0}$ is a product of the number $z=2 D$ of nearest neighbour nn spins and the constant $J_{0}$ of the single $(i-j) n n$ interaction $\left(J_{0}=J_{i j}\right.$ for $n n$ sites $i$ and $j$ ). The assumption for $n n$ interactions does not restrict the generality of the present consideration, because it is valid for a large class of short-range interactions [9]. The $\phi^{4}$-interaction part of the Hamiltonian is represented again by the corresponding term in Eq. (2), where we must substitute $\mathcal{U}$ with the interaction parameter $u_{0}$ of the form

$$
u_{0}=\frac{J^{2} T}{8 \Gamma^{3}}\left[\operatorname{th}\left(\frac{\Gamma}{T}\right)-\frac{\Gamma}{T}+\frac{\Gamma}{T} \operatorname{th}^{2}\left(\frac{\Gamma}{T}\right)\right] .
$$

In this variant of the theory, the dimensionless wave components $k_{i}$ are given by $k_{i}=2 \pi a n_{i} \tau / L_{i}$, where

$$
\tau=\sqrt{\frac{J \operatorname{th}(\Gamma / T)}{2 D \Gamma}}
$$

and $n_{i}=0, \pm 1, \ldots$ The upper cutoff $\Lambda$ of the magnitude $k\left(=\left|k_{i}\right|\right)$ of the wave vector components is defined by $\Lambda=\gamma \pi \tau$, where $\gamma$ is a small number $(\gamma \ll 1)[9]$.

All quantities in the last version of the field Hamiltonian $(\mathcal{H}=H / T)$ are dimensionless. The volume $V=$ $\left(L_{1} \ldots L_{D}\right)$ of the lattice participating in the $\phi^{4}$-part of Eq. (2) must be substituted by $\left(V / a^{D}\right)=N$ - the number of spins; hereafter we shall set $a=1$ and, hence, $V=N$. The dimensionless Matsubara frequencies $\nu_{l}$ describe the quantum effects $[1,2]$.

\section{MEAN FIELD RESULTS}

We shall reconsider the MF results [5] for the TIM because we want to clarify the properties of this model at $T=0$. Besides, we shall present a detailed comparison of the MF results for the classical $\left(T_{c}=J, \Gamma_{c}=0\right)$ and the zero-temperature $\left(T_{c}=0, \Gamma_{c}=J\right)$ critical points. Within the standard MF approximation we shall consider only the uniform mode $\phi(0)$ of the filed $\phi$. In this variant of the theory the quantum effects are ignored $\left(\nu_{l}=0\right)$.

It will be more convenient to use the MF free energy $F=T \mathcal{H}[\phi(0)]$ instead of the dimensionless MF free energy $\mathcal{H}[\phi(0)]$. This choice, after a change of the nonequilibrium order parameter from $\phi(0)$ to $\phi_{0}=$ $(T / V)^{1 / 2} \phi(0)$, will produce an extra factor $1 / T$ in front of the $\phi_{0}^{4}$-term of the free energy $F$, which makes it possible to avoid difficulties in our further analysis connected with the definition of the order parameter $\phi_{0}$ at $T=0$. The problem is related to the fact, that the parameter $u_{0}$ is proportional to $T$, see Eq. (8), and the investigation within the original parameter $\phi(0)$ will produce a result for the equilibrium order parameter of the form $\phi(0) \sim(1 / T)^{1 / 2}$ which is divergent for $T \rightarrow 0$. The Gibbs thermodynamic potential in the form 


$$
F=V\left[\frac{t_{0}}{2} \phi_{0}^{2}+\frac{u_{0}}{T} \phi_{0}^{4}\right]
$$

allows a correct MF analysis at low temperatures.

The presence of the extra factor $1 / T$ in the second term of $F$ in (10) cannot be interpreted as a manifestation of the CQC in a primitive MF variant although the CQC is usually followed by a change of the interaction constant $u$ to $v \sim(u / T)[1,2]$. The reason is that no quantum effects are present in Eq. (10). The above construction is made rather to avoid a wrong interpretation of the MF zero-temperature phase transition point $\left(T_{c}=0\right)$ as a tricritical point [2].

The analysis of the free energy (10) can be made in a straightforward way and we shall not present it in details. Moreover, a bigger part of the results, which we mention in our discussion, are known from the preceding papers (see Refs. [4,5]). Our aim is to establish a detailed picture of the LT behaviour of TIM in the MF approximation as the basis of RG studies. In order to demonstrate the specific features of the LT critical properties of TIM we shall compare them with the critical properties at the HT critical points $\left(T_{c} \sim J\right)$.

The MF phase diagram of TIM is given by $t_{0}\left(T_{c}, \Gamma\right)=$ 0 or, which is the same by $t_{0}\left(T, \Gamma_{c}\right)=0$; see the phase diagram $(T, \Gamma)$ given in Fig. 1 . The curve of the second order phase transition is given by

$$
T_{c}(\Gamma)=\frac{\Gamma}{\operatorname{Arth}\left(\frac{\Gamma}{J}\right)}
$$

or, equivalently by $\Gamma_{c}(T)$, which can be determined by solving the transcendental equation

$$
\frac{\Gamma_{c}}{J}=\operatorname{th}\left(\frac{\Gamma_{c}}{T}\right)
$$

Despite the simple form of Eq. (10) the critical properties of the TIM cannot be investigated analytically for the whole curve $T_{c}(\Gamma)$ because of the quite complex dependence of the parameters $t_{0}$ and $u_{0}$ on $T$ and $\Gamma$. Here we shall consider two limiting cases:

(i) The HT critical behaviour in the vicinity $(|T-J|<$ $J$ ) of the "classical" critical point $T_{c}(0)=J$ and near the neighbouring critical points $\left[T_{c}(\Gamma) \sim J, \Gamma \ll J\right]$.

(ii) The LT critical behaviour in the vicinity $(\mid \Gamma-$ $J \mid<J)$ of the zero-temperature critical point $\left[T_{c}(J)=\right.$ $\left.0, \Gamma_{c}(0)=J\right]$ and the neighbouring critical points with coordinates $T_{c} \ll J$ and $\Gamma_{c} \sim J$.

The four typical ways of crossing of the phase transition curve in the HT and LT regimes are shown in Fig. 2. We shall investigate the critical behaviour of the HT critical point $\Gamma_{c}=0$ along the HT lines $0-J$ and $A-J$ as well as the critical behaviour near a neighbour critical point $\Gamma_{c} \sim 0$ along the HT lines $a-a$ and $b-b$ (Fig. 2). In similar way, four thermodynamic processes along the LT lines $0_{-}^{-}$ $J, A-J, a-a$, and $b-b$, as shown in Fig. 2, will be discussed for the zero-temperature critical point $\left(T_{c}=0, \Gamma_{c}=J\right)$ and for neighbouring LT critical points $\left(T_{c} \sim 0, \Gamma_{c} \sim J\right)$.

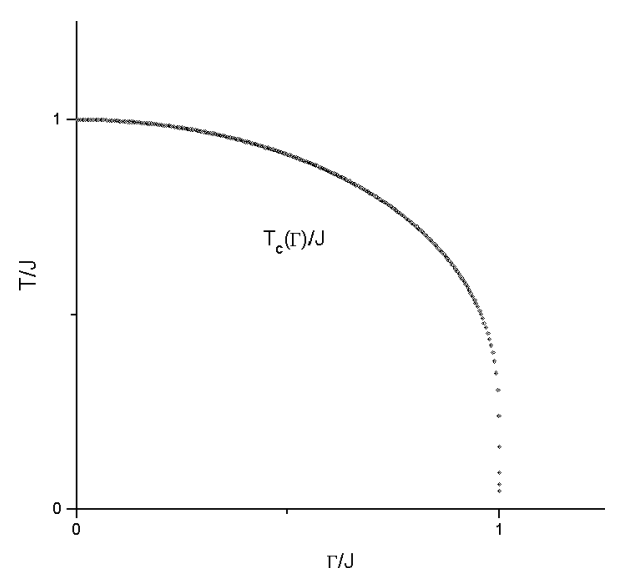

Fig. 1. The graphical representation of Eq. (11) for $J=1$.

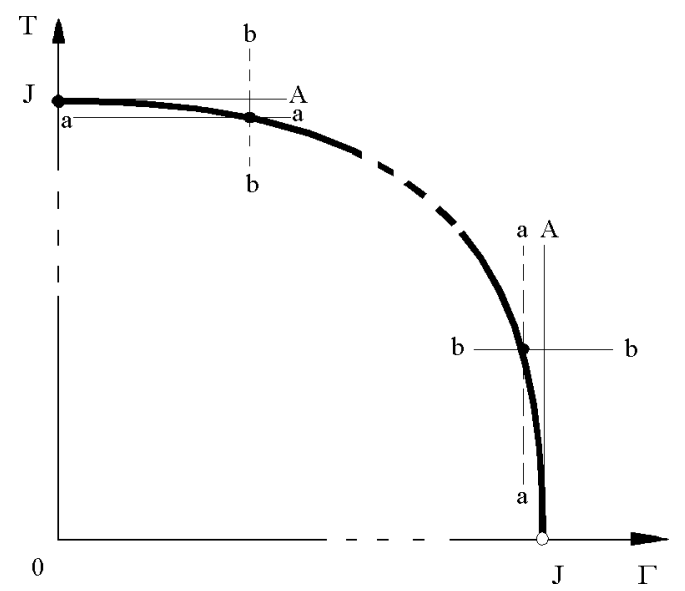

Fig. 2. The HT and LT regions of the phase diagram. The notations are explained in the text.

The intermediate critical points, say, $\left(T_{c} \sim \Gamma_{c}\right)$ can be investigated only by a numerical analysis. There exists a smooth crossover between the classical $\left(T_{c}=J\right)$ and the zero-temperature $\left(T_{c}=0\right)$ critical regimes when the parameter $\Gamma$ varies from zero to $J$.

\section{A. High Temperature Properties}

In this regime we consider the part of the phase diagram (Figs. 1 and 2), where $T \gg \Gamma$.

\section{Line $0-J$}

The critical behaviour along the line $\Gamma=0$ completely coincides with that of the classical Ising model in the MF approximation [2,9]. In this case the parameters $t_{0}$ and $u_{0}$ in (10) take the usual values $t_{0}=(T-J) / T$ and $u_{0}=J^{2} / 12 T^{2}[2,9]$. 


\section{Line $b-b$}

The shape of the curve $T_{c}(\Gamma)$ for $\Gamma \sim 0$ is given by $T_{c}(\Gamma) \approx J-\Gamma^{2} / 3 T^{2}$. The magnetic susceptibility $\chi_{T}$ for $\Gamma \sim 0$ is

$$
\chi_{T} \sim \frac{T_{c}}{\left|T-T_{c}\right|}\left[1+0\left(\Gamma^{4} / T_{c}^{4}\right)\right] .
$$

The corrections $0\left(\Gamma^{2} / T_{c}^{2}\right)$ are irrelevant. The suffix " $T$ " of $\chi_{T}$ denotes that the main dependence of the susceptibility is on the temperature $T$. The critical exponent $\gamma_{T}$ of the susceptibility with respect to variations of $T$ is $\gamma_{T}=1$. The order parameter has also a classical (MF) behaviour, $|\phi|^{2} \sim\left(T_{c}-T\right)$, described by the critical exponent $\beta_{T}=1 / 2$ and negligible corrections $0\left(\Gamma^{2} / T_{c}^{2}\right)$ to the main $T$-dependence. In general, this is the usual MF behaviour ( $\S$ III.A.1) of the classical Ising model $(\Gamma \equiv 0)$ with small $\Gamma$-corrections $0\left(\Gamma^{2} / T_{c}^{2}\right)$.

\section{Line $A-J$}

The critical behaviour along the line $A-J$ given by $T=J$ is described by

$$
t_{0}=\frac{1}{3}\left(\frac{\Gamma}{J}\right)^{2}+0\left(\Gamma^{4} / J^{4}\right)
$$

and, hence, the magnetic susceptibility $\chi_{\Gamma}^{(>)}$in the paramagnetic phase $\left(\phi_{0}=0\right)$ is $\chi_{T}^{(>)}=t_{0}=3 J^{2} / \Gamma^{2}$. The suffix " $\Gamma$ " in $\chi_{\Gamma}^{(>)}$denotes that the susceptibility is calculated for variations of the parameter $\Gamma$ at fixed $T$ and the superscript ">" denotes that the calculated value of this quantity is only valid in the para-phase $\left(\phi_{0}=0\right)$. The result for the susceptibility $\chi_{\Gamma}^{(>)}$obtained above yields the critical exponent $\gamma_{\Gamma}^{(>)}=2$. The value $\gamma_{\Gamma}^{(>)}=2$ corresponds to the universality class of the three dimensional $(D=3)$ spherical model [2].

\section{Line aa}

Along the HT line $a-a$, where $T \sim J, u_{0}=J^{2} / 12 T^{2} \approx$ $1 / 12$, we obtain

$$
\left|\phi_{0}\right|^{2} \sim \chi^{-1} \sim\left|t_{0}\right|
$$

where

$$
\chi \approx \frac{3 T_{c}^{3}}{2 J \Gamma_{c}} \frac{1}{\left|\Gamma-\Gamma_{c}\right|} \approx \frac{3 J^{2}}{2 \Gamma_{c}} \frac{1}{\left|\Gamma-\Gamma_{c}\right|}
$$

for $\left(\Gamma_{c} \rightarrow 0^{+}\right)$. This result corresponds to the usual MF behaviour $\left(\gamma_{\Gamma}=1, \beta_{\Gamma}=1 / 2\right)$ of the Ising model, but the scaling amplitudes are very large $\left(\Gamma^{-1} \sim \infty\right)$. For this reason the critical behaviour along the line a $a$ is quite unusual. For $\Gamma \gg \Gamma_{c}$ and $\Gamma_{c} \rightarrow 0$, this behaviour smoothly tends to the critical behaviour explained in $\S$ III.A.3.

It can be concluded that the behaviour along the HT lines $0-J$ and $b-b$ belongs to the usual MF predictions whereas the MF critical behaviour along the HT lines $A-J$ and $a-a$ is quite special. The latter has a similarity with the critical behaviour predicted by the spherical approximation for the Ising model [2].

\section{B. Low Temperature Properties}

Here we consider the domain $T \ll \Gamma$ of the phase diagram shown in Figs. 1 and 2.

\section{Line $0-J$}

We obtain $t_{0} \approx(\Gamma-J) / \Gamma, \phi_{0} \sim\left|t_{0}\right|^{1 / 2}, \chi_{\Gamma} \sim \Gamma /|\Gamma-J|$ and, therefore, $\gamma_{\Gamma}=1$ and $\beta_{\Gamma}=1 / 2$. There exists a full coincidence between the standard MF behaviour with respect to variations of $T$ around $J$ at $\Gamma=0$ (see $\S$ III.A.1) and the present critical behaviour due to variations of $\Gamma$ around $J$ at $T=0$. This correspondence can be written as $T \leftrightarrow \Gamma$.

\section{Line $b b$}

The parameter $t_{0}$ is

$$
t_{0} \approx \frac{J\left(\Gamma-\Gamma_{c}\right)}{\Gamma_{c}^{2}}\left(1-\frac{4 \Gamma_{c}}{T} e^{-2 \Gamma_{c} / T}+\ldots\right)
$$

Here $t_{0}$ tends to the value $(\Gamma-J) / \Gamma$ when $\Gamma_{c}(T) \rightarrow J$ for $T \rightarrow 0$; see $\S$ III.B.1. The critical exponents are the same as those given in $\S$ III.B.1.

\section{Line $A-J$}

As in $\S$ III.A.3 there is no ordering along the line $A-J$. However, the other features of the transition along this line are quite different from those presented in $\S$ III.A.3. The susceptibility above the zero-temperature critical point $\left(\Gamma_{c}=J\right)$ is

$$
\chi_{T}^{(>)}=\frac{1}{2} e^{2 J / T}
$$

and, hence, $\gamma_{T}^{(>)}=\infty$. This behaviour is quite different from that along the HT line $A-J$ (§ III.A.3). The reason is in the different behaviour of the functions $t_{0}(T, \Gamma)$ and $u_{0}(T, \Gamma)$ in the HT and LT regimes. 


\section{Line aa}

Along the line $a-a$

$$
t_{0} \approx \frac{4 J\left(T-T_{c}\right)}{T_{c}^{2}} e^{-2 \Gamma / T_{c}}
$$

and, therefore, for both $T<T_{c}$ and $T>T_{c}, \chi_{T} \sim$ $1 /\left|t_{0}\right|$, and $\gamma_{T}=1$. However, the amplitude $\chi^{(0)} \sim$ $\left(T T_{c}\right) \exp \left(2 \Gamma / T_{c}\right)$ of the scaling relation $\chi_{T}=\chi^{(0)} /(T-$ $T_{c}$ ) exponentially increases at $T_{c} \rightarrow 0$ and this is the manifestation of an unusual behaviour that might be of interest to experiments.

There exists a smooth transition from this behaviour to the exponential behaviour mentioned in $\S$ III.B.3. In order to obtain (19) from (18) one should consider that $J\left|T-T_{c}\right| \gg T_{c}^{2}$. As $\left(u_{0} / T\right) \approx J^{2} / 8 \Gamma^{3}$ for $(\Gamma \sim J \gg T)$, we have $\phi_{0} \sim\left|t_{0}\right|^{1 / 2}$ with $t_{0}$ given by (19), which means that the order parameter exponent has the classical value $\beta_{T}=1 / 2$, but the scaling amplitude $\left[\sim T_{c}^{-1} \exp \left(-\Gamma / T_{c}\right)\right]$ of the order parameter exponentially decreases for $T_{c} \rightarrow 0$.

The critical properties along the HT and LT lines $a-a$ have a similarity because they have equal critical exponents and large amplitudes of the scaling laws. But the difference between these cases is in the form of the large scaling amplitudes. While the HT scaling amplitudes are divergent at the critical points by power laws, the same divergency in the LT case is given by exponential laws.

\section{Comparison of High and Low Temperature Properties. Crossover from the Former to the Latter}

In general, there are similarities between the MF critical properties at HT and LT. The MF behaviour along the HT and LT lines $0-J$ are the same. Along the lines $b-b$, the critical properties are the same, too apart from the form of unessential corrections. The critical properties along the HT and LT lines $a-a$ are different owing to the form of the scaling amplitudes. While the HT scaling amplitudes are divergent at the critical points by power laws, the divergency of the scaling amplitudes in the LT case is given by exponential laws. The most essential difference between the critical properties at HT and LT occurs along the lines $J-A$. The critical properties on these lines are quite unusual.

The differences between the HT and LT properties of TIM mean that there is a "high-to-low temperature" crossover (HLTC) in the properties of the phase transitions in TIM. This picture has been outlined within the MF approximation, in which the quantum effects are neglected. As we shall see in the next Section there are large domains in the LT region of the phase diagram where the quantum effects are irrelevant and the classical description is consistent. Besides, we shall show by the GC that the MF results are valid in broad domains around of the phase transition curve $T_{c}(\Gamma)$.

\section{GINZBURG CRITERION}

The $(T, \Gamma)$ domains of validity of the MF results in Section III can be investigated by the Ginzburg criterion (GC) [2]. Here we shall use a standard derivation of this criterion from the first-order perturbation contribution to the "self-energy" $t_{0}[2]$ :

$$
\tilde{t}_{0}=t_{0}+12 u_{0} A_{1}\left(t_{0}\right)
$$

with

$$
A_{1}\left(t_{0}\right)=\int \frac{d^{D} k}{(2 \pi)^{D}} S_{1}\left(t_{0}, k\right),
$$

where

$$
S_{1}\left(t_{0}, k\right)=\frac{\Gamma \operatorname{cth}\left(\frac{\Gamma}{T} \sqrt{k^{2}+t_{0}}\right)}{T \sqrt{k^{2}+t_{0}}} .
$$

The perturbation insertion $\left[12 A_{1}(0)\right]$ to the coordinates of the critical points $\left(T_{c}, \Gamma_{c}\right)$ will be neglected as being irrelevant to the present discussion. The GC for the validity of the MF results is given in the general form [2]:

$$
\left|t_{0}\right|>12 u_{0}\left[A_{1}(0)-A_{1}\left(t_{0}\right)\right]
$$

The GC (23) is valid for both the paramagnetic $\left(t_{0}>0\right)$ and the ferromagnetic $\left(t_{0}<0\right)$ phases; in the ferromagnetic phase the factor $1 / 2$ should be added to the r.h.s. of (23).

Because of the difficulties in the analytical treatment of the integral (21) for any $T$ and $\Gamma$, we shall consider two limiting cases:

$$
\frac{\Gamma}{T} \sqrt{t_{0}} \ll 1
$$

and

$$
\frac{\Gamma}{T} \sqrt{t_{0}} \gg 1
$$

As the small wave numbers $(k \sim 0)$ give the main contribution to the integral (21) near the critical points $\left(t_{0} \sim 0\right)$, the limiting cases $(24)$ and (25) determine the two main critical regimes: the classical critical regime corresponding to the case (24) and the quantum critical regime corresponding to the case (25).

Inequality (24) can be written by the two relevant lengths in TIM, the correlation length $\xi=1 /\left|t_{0}\right|^{1 / 2}$ of the thermal (classical) fluctuations, and the thermal wave length $\lambda_{T} \sim(\Gamma / T)$ [2] of the quantum correlations (sometimes referred to as quantum fluctuations). Thus the inequality (24) means that $\lambda_{T} \ll \xi$ and, hence, 
that the quantum effects are irrelevant for the quasimacroscopic critical behaviour in the corresponding domain of the plane $(T, \Gamma)$. The inequality (25) means, that the quantum correlations with the characteristic length $\lambda_{T} \gg \xi$ may drastically change the critical behaviour in certain LT and HT domains of the plane $(T, \Gamma)$. An important difference between the classical and the quantum critical properties, namely, the effective change of the spatial dimensionality of the fluctuation modes $\phi(q)$, is described by the CQC mentioned in Section I.

For HT $(T \gg \Gamma)$, the inequality $(24)$ is always fulfilled (remember, that $t_{0}<1$ ). This means that the classical domain in the $(T, \Gamma)$ plane defined by (24) covers the whole HT domain. As we shall see, it covers also a part of the LT domain $(T \ll \Gamma)$. In the LT domain $(T \ll \Gamma)$ the critical behaviour can be either a classical behaviour, namely, corresponding to the inequality (24), or, a quantum behaviour corresponding to $(25)$. This means that the LT phase transitions in TIM can exhibit both classical and quantum critical phenomena. The CQC crossover does not occur for all critical phenomena in the LT domain, as it will be demonstrated in the remainder of this paper.

The classical critical phenomena are considered by the classical (usual) version of the GC which will be referred to as CGC. In contrast, the quantum critical phenomena should be treated by the quantum version of the same criterion which will be referred to as QGC.

The MF picture of the critical behaviour outlined in Section III do not include quantum effects. It is a product of classical phenomena only. Having in mind this circumstance it seems that the consistent investigation of the domains of validity of the MF results along all HT and LT lines considered in Section III should be made by the CGC, where quantum effects are not taken into account, too. While this is the systematic approach to the problem, it must be emphasized that the CGC is valid only under condition (24). Therefore, we must derive and apply the QGC to the MF behaviour in certain LT cases, in which the condition (24) is not satisfied (see $§$ IV.A.2 and Section IV.B). Note, that the wrong choice of the GC leads to a wrong conclusion about the lack of any $(T, \Gamma)$ domain of validity of the MF results or about improper restrictions on the validity of these results (see $\S$ IV.A.2 and $\S$ IV.B.1).

\section{A. Classical Ginzburg Criterion}

Using the inequality (24) and performing standard calculations with the help of Eqs. (21)-(23) we obtain the CGC

$$
\left|t_{0}\right|^{(4-D) / 2}>12 u_{0} C_{D}
$$

where $2<D<4, C_{D}=K_{D} \Gamma(1-D / 2) \Gamma(2-D / 2)$ with $K_{D}=1 / 2^{D-1} \pi^{D / 2} \Gamma(D / 2)$. The only integer dimensionality $D$ between the upper critical dimensionality $D_{U}=4$ and the lower critical dimensionality $D_{L}=2$ is $D=3$. For the sake of simplicity we shall consider $3 D$ systems, i.e. systems of dimensionality $D=3$.

For $D=3$, the CGC (26) becomes

$$
\left|t_{0}\right|>\left(\frac{6}{\pi}\right)^{2} u_{0}^{2}
$$

For HT, this CGC takes the form

$$
\left|t_{0}\right|>\frac{J^{4}}{4 \pi^{2} T^{4}}
$$

where we have used the HT value $u_{0}=\left(J^{2} / 12 T^{2}\right)$. For LT the CGC (27) reads

$$
\left|t_{0}\right|>\left(\frac{3}{4 \pi}\right)^{2} \frac{J^{4} T^{2}}{\Gamma^{6}}
$$

where the LT value $u_{0}=J^{2} T / 8 \Gamma^{3}$ has been used; see Eq. (8) for $T \ll \Gamma$. We shall neglect terms of order $0\left(\left|T-T_{c}\right|^{2}\right)$ and $0\left(\left|\Gamma-\Gamma_{c}\right|^{2}\right)$. Moreover, factors of type $(J / T)$ in the r.h.s. of inequalities corresponding to HT will be often set equal to unity. The same will be done for factors of type $(\Gamma / J)$ and $\left(\Gamma_{c} / J\right)$ in the r.h.s. of inequalities corresponding to LT. For example, in $(28)$ we can set $(J / T)^{4} \approx 1$, and in $(29)$ the factor $(J / \Gamma)^{4}$ can also be equalized to unity. All these approximations are consistent with the scope of validity of the Landau expansion in powers of the order parameter.

\section{Classical Ginzburg Criterion for High Temperatures}

Using Eqs. (13) and (28) we obtain that the MF results along the HT line $b-b$ are valid for

$$
\left|T-T_{c}\right|>0.025(J / T)^{4} T_{c}
$$

One should add to this condition the requirement for the validity of the Landau expansion in powers of the order parameter $\phi[2]$, namely $\left|T-T_{c}\right|<T_{c}$. By setting $T_{c}=J$ in (30) one obtains the CGC along the HT line $0-J(\S$ III.A.1).

In the same way one may use Eqs. (14) and (28) in order to show that the CGC along the HT line $A-J$ will be: $\Gamma>0.3 J$. The latter yields quite a strong restriction in view of the fact that the HT condition is $\Gamma \ll J$. For the HT line $a-a$ we have the criterion

$$
\left|\Gamma-\Gamma_{c}\right|>0.04 J^{3} / \Gamma_{c} T
$$

where $\left(J^{3} / \Gamma_{c} T\right) \sim\left(J^{2} / \Gamma_{c}\right)$. In this case the applicability of the MF results is also quite restricted because of the additional conditions $J \sim \Gamma \gg \Gamma_{c} \sim \Gamma$.

It can be concluded that the MF results for the HT critical behaviour produced by $T$-variations around the value $T \sim J$ at fixed $\Gamma \ll J$ have a well established 
broad region of validity outside the Ginzburg critical region. However, the validity of the HT MF results related with variations of the parameter $\Gamma$ at fixed $T(\sim J)$ is quite restricted.

\section{Classical Ginzburg Criterion for Low Temperatures}

Along the line $0-J$, the temperature is equal to zero and, hence, condition (24) cannot be fulfilled. Therefore, the CGC cannot be applied to this case. The critical behaviour along the line $0-J$ does not belong to the classical domain and the region of validity of the $\mathrm{MF}$ results in $\S$ III.B.1 should be determined by the QGC (Section IV.B).

For the critical behaviour along the LT line $b-b$ condition (24) is fulfilled for

$$
\left|\Gamma-\Gamma_{c}\right| \ll\left(\frac{\Gamma_{e}}{\Gamma}\right)^{2} \frac{T^{2}}{J}
$$

where $\Gamma_{c} \sim \Gamma \sim J$, and $J \gg T$. The criterion (29) yields

$$
\left|\Gamma-\Gamma_{c}\right|>0.06 \frac{J^{3} T^{2}}{\Gamma^{4}}
$$

Conditions (32) and (33) can be satisfied only for $\Gamma \gg$ $(J / 2)$, which is a very strong restriction. The latter simply means that the criterion (33) does not work for LT critical points $\left(\Gamma \sim \Gamma_{c} \sim J\right)$. The correct criterion is deduced in $\S$ IV.B.1 with the help of the QGC.

The MF critical behaviour shown by the LT line $A-J$ is valid for

$$
\frac{J}{T}>0.17 e^{J / T}
$$

whereas the CGC for the LT line $a-a$ is

$$
\left|T-T_{c}\right|>0.01\left(\frac{T_{c}^{4}}{J^{3}}\right) e^{2 J / T_{c}}
$$

Conditions (34) and (35) present quite restricted regions of validity of the corresponding MF results.

\section{B. Quantum Ginzburg Criterion}

If condition (25) is fulfilled, Eqs. (21) and (22) will yield

$$
A_{1}\left(t_{0}\right)=\frac{\Gamma}{T} \int \frac{d^{D} k}{(2 \pi)^{D}} \frac{1}{\sqrt{k^{2}+t_{0}}} .
$$

Criterion (23) takes the form

$$
\left|t_{0}\right|>12 u_{0} K_{D} \int d k \cdot k^{D-1}\left(\frac{1}{k}-\frac{1}{\sqrt{k^{2}+t_{0}}}\right) \text {, }
$$

where $1<D<3$ and $v_{0}=(\Gamma / T) u_{0}$. As the quantum correlations are taken into account in criterion (37), we obtain that the quantum lower critical dimensionality is $D_{L}^{(q)}=1=\left(D_{L}-1\right)$ and that the quantum upper critical dimensionality is $D_{U}^{(q)}=3=\left(D_{U}-1\right)$. The last relationships between the critical dimensionalities in the classical and the quantum regimes is a manifestation of the $\mathrm{CQC}: D \rightarrow(D+1)$.

$$
\text { 1. } D=2
$$

In order to avoid an unnecessary complexity in our analysis we shall focus our attention in the case of $2 D$ systems $(D=2)$. We calculate integral (37) for $\Lambda=\infty$ at $D=2$ and obtain the QGC in the form

$$
\left|t_{0}\right|>\left(\frac{6}{\pi}\right)^{2} v_{0}^{2}
$$

c.f. Eq. (27). Having in mind that for LT $v_{0}=J^{2} / 8 \Gamma^{2}$, we obtain

$$
\left|t_{0}\right|>\left(\frac{3}{4 \pi}\right)^{2}\left(\frac{J}{\Gamma}\right)^{4}
$$

The criterion (39) cannot be applied to the behaviour along the LT line $J-A$, where inequality (25) is not valid for $J \gg T$. Along the LT line $a-a$, inequality (25) yields

$$
\left|T-T_{c}\right| \gg \frac{T_{c}^{4}}{4 J^{3}} e^{2 J / T_{c}}
$$

This condition combined with the Landau condition $\left|T-T_{c}\right|<T_{c}$ and the LT condition $(T \ll \Gamma)$ yields that there is not any $(T, \Gamma)$ domain of applicability of the QGC to the critical behaviour along the LT line $a-a$.

Along the line $b-b$ we obtain the QGC in the form

$$
\left|\Gamma-\Gamma_{c}\right|>0.06 \Gamma_{c}\left(\frac{J}{\Gamma_{c}}\right)^{3} \sim 10^{-2} \Gamma_{c}
$$

Setting $\Gamma_{c}=J$ in this criterion we find the QGC along the LT line $0-J$. Because of the very sharp slope of the transition curve $T_{c}(\Gamma)$ near $\Gamma=J$, the QG criterions along the lines $b-b$ and $0-J$ are practically the same. The Ginzburg critical region $\left(\sim 10^{-2} \Gamma_{c}\right)$ given by (41) is relatively large; c.f. (30).

The quantum condition (25) along the LT line $b-b$ can be written in the simple form $J\left(\left|\Gamma-\Gamma_{c}\right|\right) \gg T^{2}$ provided one sets $\left(\Gamma / \Gamma_{c}\right)^{2} \approx 1$. As $J \gg T$, the condition becomes

$$
\left|\Gamma-\Gamma_{e}\right|>T
$$


which is obviously consistent with (41). Condition (42) shows that the quantum region approaches the critical point $\Gamma_{c}$ when $T$ decreases to zero [1]. On the one hand, the whole surrounding of the zero-temperature critical point $\left(\Gamma_{c}=J\right)$ is influenced by the quantum effects which produce the CQC. On the other hand, however, criterion (41) shows that the size of the critical region does not tend to zero for $T \rightarrow 0$. Therefore, the "quantum" critical behaviour $(T=0)$ is a result of the usual classical fluctuations which, owing to the quantum effect of the dimensional crossover, act in $(D+1)$ rather than in $D$ spatial dimensionalities. There are no new (quantum) critical modes but rather $(D+1)$-dimensional classical (thermal) fluctuations. The latter exist as strongly interacting modes for any $T \geq 0$ provided $\Gamma_{c} \sim J>0$; see (41).

\section{Discussion}

The application of the CGC to HT has been shortly discussed in the end of $\S$ IV.A.1. It has been shown in $\S$ IV.A.2 that the CGC cannot be applied to the phase transitions along the lines LT $0-J$ and $b-b$ because these transitions belong to the quantum regime, where the CGC is not valid. The quantum effects manifest themselves in the properties of the phase transitions along the mentioned lines and this is the single case of a quantum critical behaviour in TIM. The latter is a pure classical behaviour in $(D+1)$ rather than $D$ spatial dimensionalities. The same classical behaviour has been investigated within the MF approximation (Section III), where quantum effects are not present. Therefore, in $\S$ IV.B.1 we have tested the classical MF results for the phase transitions in the quantum regime by the QGC and we have confirmed that these results have a region of validity near the LT phase transition points. The investigation has been based on the fact that the MF results do not include quantum effects and that they should be tested with those versions of the $\mathrm{GC}$ which are valid in the corresponding domains of the $(T, \Gamma)$ plane. In this way we have obtained well established regions of validity of the MF behaviour predicted for the "quantum transitions" along the LT lines $0-J$ and $b-b$.

Alternatively, the QGC cannot be applied to the phase transitions along the LT lines $J-A$ and $a-a$, because these transitions belong to the classical region. Although the line $a-a$ can be situated very near to the line $J-A$ and, hence, the phase transition temperature $T_{c}$ can be very near to zero, the transition along this line remains in the classical domain because of the very sharp slope of the phase transition curve $T_{c}(\Gamma)$ in a close vicinity of $\Gamma_{c}=J$.

Finally, we note, that the MF results for the properties of the HT transitions driven by $\Gamma$-variations and of the LT transitions driven by $T$-variations (lines $A-J$ and $a-a)$ have more narrow regions of validity than the MF results for the corresponding phase transitions along the lines $0-J$ and $b-b$.

\section{RENORMALIZATION GROUP ARGUMENTS}

The RG recursion relation [2] for the interaction parameter $u_{0}$ in the one-loop approximation is

$$
u_{0}^{\prime}=b^{4-D} u_{0}\left[1-36 u_{0} A_{2}(0, b)\right]
$$

where $b>1$ is the RG rescaling factor and

$$
A_{2}\left(t_{0}, b\right)=-\frac{\partial A_{1}\left(t_{0}, b\right)}{\partial t_{0}}
$$

In Eq. (44) $A_{1}\left(t_{0}, b\right)$ is the integral (21) with lower $\left(0<b^{-1}<1\right)$ and upper $(\Lambda=1)$ cutoffs of the wave number $k$.

The other recursion relations are

$$
t_{0}^{\prime}=b^{4-D}\left[t_{0}+12 u_{0} A_{1}\left(t_{0}, b\right)\right]
$$

and

$$
\left(\frac{\Gamma}{T}\right)^{\prime}=b^{z}\left(\frac{\Gamma}{T}\right),
$$

where the dynamical critical exponent $z$ is equal to unity in this order of the theory $(z=1)$.

\section{A. Classical Renormalization Group}

If condition (24) of the classical behaviour is valid, the integral $A_{2}(0, b)$ has a logarithmic infrared divergence at the upper critical dimensionality $D_{U}=4$. For $D=4$, $A_{2}(0, b)=K_{D} \ln b$. Further, using the standard RG analysis [1] one reveals the usual universality class of the critical behaviour of the classical Ising model $(\Gamma=0)$. Thus one can prove that the asymptotic critical behaviour in the Ginzburg critical region along the HT lines $0-J$ and $b-b$ will belong to the nontrivial Ising class of universality $(1, D)$ rather than to the simple MF behaviour. The critical behaviour is described by the familiar $\epsilon=(4-D)-$ corrections to the MF critical exponents [2].

Under the same classical condition (24), the RG yields a nontrivial critical behaviour for all HT phase transitions. The behaviour along the HT lines $0-J$ and $b-b$ belongs to the Ising universality class $(1, D)$, but the other HT transitions exhibit new unusual properties. In order to clarify this point it is more convenient to define the distance from any critical point $\left(T_{c}, \Gamma_{c}\right)$ by the parameter $t_{0}$ rather than by the differences $\left|T-T_{c}\right|$ and $\left|\Gamma-\Gamma_{c}\right|$. In this way the critical exponents will be defined as exponents describing scaling laws with respect to the quantity $t_{0}$. Under this stipulation, the classical condition (24) and the corresponding RG analysis lead to the familiar critical exponents of the universality class $(1, D)$ for all phase transitions for which the HT condition $(T \gg \Gamma)$ is also satisfied. The scaling laws in powers 
of $t_{0}$ will have the $\epsilon=(4-D)$-corrections corresponding to the universality class $(1, D)$. The real critical behaviour in terms of $\left|T-T_{c}\right|$ or $\left|\Gamma-\Gamma_{c}\right|$ will be determined by the latter result and the MF prediction for the function $t_{0}(T, \Gamma)$. For example, the values the MF spherical exponents deduced for the behaviour along the HT line $A-J$ will acquire standard $\epsilon$-corrections. This is a quite unexpected prediction about the critical behaviour along the HT line $A-J$. Along the HT line $b-b$ we obtain a standard $(1, D)$-behaviour with large scaling amplitudes.

The LT behaviour along the LT lines $A-J$ and $a-a$ satisfies the classical condition (24). In this case we must use the classical RG equations with the upper dimensionality $D_{U}=4$, but the (bare) parameters $t_{0}$ and $u_{0}$ should be taken with their LT values. In particular we must use the value $u_{0}=v_{0}(T / \Gamma)$, where $v_{0}=J^{2} / 8 \Gamma^{2}$. Although $u_{0} \rightarrow 0$ for $T \rightarrow 0$, the formal solution of the RG equations will yield again the universality class $(1, D)$ for all dimensionalities $2<D<4$ and $T>0$. Therefore, the exponential dependence explained in $\S$ III.B.3 will be modified by $\epsilon$-corrections to the numerical exponential factor $(-2)$ in $t_{0}$. The $\epsilon$-corrections can be easily found by using $t_{0}=2 \exp (-2 J / T)$ and performing the standard RG calculation of the critical exponent $\gamma$ [2]. In the same way one may perform the RG treatment along the LT line $a-a$, where the universality class $(1, D)$ is characterized with anomalously large scaling amplitudes. In this case, the critical behaviour remains within the universality class $(1, D)$.

\section{B. Quantum variant of Renormalization Group}

The critical behaviour along the LT lines $0-J$ and $b-b$ is described by the quantum version of the RG. The reason is that the quantum condition (25) should be applied for this case. It is not difficult to show that the RG Eq. (43) should be transformed to [1,2]

$$
v_{0}^{\prime}=b^{3-D} v_{0}\left[1-36 v_{0} K_{3} \ln b\right]
$$

Now the RG equations are written in terms of parameters $t_{0}$ and $v_{0}$. The upper quantum critical dimensionality is $D_{U}^{(q)}=3$ and the asymptotic critical behaviour for dimensionalities $D<3$ belongs to the Ising universality class $(1, D+1)$. This is the RG proof of the CQC [1]. The proof is valid in that LT region of the phase dia$\operatorname{gram}(T, \Gamma)$, where both the LT condition $(T \ll \Gamma)$ and the quantum condition (25) are fulfilled.

Remember inequality (42). It means that, in a strict mathematical sense, the CQC occurs only along the zero temperature line $0-J$ which is practically inaccessible for experiments. This phenomenon is valid only for the phase transition at the zero-temperature critical point $\left(T_{c}=0, \Gamma_{c}=J\right)$, provided the latter is crossed by $\Gamma-$ variations at $T=0$. The Ginzburg region $\left(\sim 10^{-2} J\right)$ around the quantum critical point remains finite and even relatively large; see (41). This means that the CQC describes classical fluctuations at $T=0$ for all values
$J>0$ of the exchange parameter. At $T=0$, owing to the inequality (41) which leads to the CQC, these well known classical fluctuations are effectively described in a "classical" $(D+1)$-dimensional system. The same picture has been shown with the help of the GC (Section III).

Along the LT line $b-b$ there is a narrow region around the critical point $\left(T_{c} \sim 0, \Gamma_{c} \sim J\right)$, where the condition (25) breaks down; see inequality (42). In this narrow region $(\sim T)$ the behaviour is given by the standard Ising class $(1, D)$ and the CQC does not occur. However, the onset of the CQC could be observed by experiments at extremely low temperatures. These temperatures can be evaluated by the conditions (41) and (42):

$$
T \ll 10^{-2} \Gamma_{\epsilon} \sim 10^{-2} J
$$

\section{Notes}

The application of the classical variant of $\mathrm{RG}$ to the zero-temperature quantum critical point $T_{c}=0$ will give a trivial (Gaussian) critical behaviour for all dimensionalities $D>D_{L}=2$. The reason is that within this classical variant of the theory, the $L T$ condition $T \ll \Gamma$ yields $u_{0} \sim T$ and, simultaneously, because of the classical condition (24), the resulting RG Eq. (43) for $u_{0}$ cannot be transformed to an equation for $v_{0}$. For $T \rightarrow 0$, the initial (bare) Hamiltonian parameters will be located on the line $u_{0}=0$ in the parameter space $\left(t_{0}, u_{0}\right)$. Therefore, the RG trajectories with such initial coordinates will not tend to the nontrivial Ising fixed point $\left(t^{I} \neq 0, u^{I} \neq 0\right)$ but will be rather attracted by the trivial Gaussian fixed point $\left(t^{G}=u^{G}=0\right)$ [2]. This leads to a prediction for a validity of the MF approximation for the zero-temperature critical behaviour ( $§$ III.B.1) for dimensionalities $D>2$.

Our consideration shows that the application of the classical variant of $\mathrm{RG}$ to the zero-temperature critical point is wrong in principle but, as we see, it gives a correct prediction for $3 D$ systems. In fact, the CQC means that at $T=0$, the $3 D$ TIM exhibits a behaviour equivalent to that of the $(3+1)$-dimensional classical Ising model. The latter is exactly the MF behaviour (apart from logarithmic corrections to the MF scaling) [2]. For $2 \mathrm{D}$ magnets, the above classical version of RG gives completely wrong results.

\section{A generalization}

The effective change of the dimensionality of TIM in the quantum critical regime is given by the dynamical exponent $z=1$ [1]. One may write the CQC as $D \rightarrow(D+z)$. This result is produced in the classical and quantum limits of Eq. (22). Instead of performing the summation over the Matsubara frequencies $\nu_{l}$ in the sum 


$$
S_{1}\left(t_{0}, k\right)=\sum_{\nu_{l}} \frac{1}{t_{0}+k^{2}+\nu_{l}^{2}}
$$

we may substitute this summation in an approximate way by an integration in dimensionalities $\delta \in[0,1]$. The idea has been proposed in preceding papers on thin films [10] and disordered Bose systems (see A. M. J. Schakel (1997) in Ref. [3]). Following this idea we shall substitute sum (49) with the integral

$$
\tilde{S}_{1}\left(t_{0}, k\right)=\left(\frac{2 \Gamma}{T}\right)^{\delta} \int \frac{d^{\delta} \nu}{(2 \pi)^{\delta}} \frac{1}{t_{0}+k^{2}+\nu^{2}}
$$

The factor $(2 \Gamma / T)^{\delta}$ is introduced because the frequency $\nu$ is proportional to the ratio $(T / 2 \Gamma)$. The straightforward calculation of the integral $\tilde{S}_{1}$ yields

$$
\tilde{S}_{1}\left(t_{0}, k\right)=\frac{\Gamma(1-\delta / 2)}{(2 \sqrt{\pi})^{\delta}}\left(\frac{2 \Gamma}{T}\right)^{\delta}
$$

For $\delta \rightarrow 0$ (the classical limit) and $\delta \rightarrow 1$ (the quantum limit) $\tilde{S}_{1}$ coincides with the exact sum $S_{1}$ from Eq. (22). The correspondence $\tilde{S}_{1} \leftrightarrow S_{1}$ for the intermediate cases $(0<\delta<1)$ of a "partial quantum effect" is approximate $[10]$.

With the help of this construction one may repeat the RG calculations and show that the $\mathrm{CQC}$ can be defined by the generalized rule $D \rightarrow(D+\delta)$, where the partial dimensionality $\delta$ varies from zero to unity. The RG results for the critical behaviour are given by a single $\tilde{\epsilon}=[4-(D+\delta)]$-expansion instead of two expansions in: $\epsilon=(4-D)$ (for the classical case) and $\epsilon_{0}=(3-D)$ (for the quantum case). The critical behaviour for any $\delta$ is described by the usual critical exponents for the Ising universality class provided the parameter $\epsilon=4-D$ is changed with $\tilde{\epsilon}=4-(D+\delta)$.

Certainly, there is a fundamental dependence of $\delta$ on $\left(\lambda_{T} / \xi\right)$ such that the function $\delta\left(\lambda_{T} / \xi\right)$ smoothly varies from zero to unity for variations of the ratio $\left(\lambda_{T} / \xi\right)$ from zero to infinity, respectively. A similar dependence has been discussed in connection with the problem for the finite-size crossover in pure and impure thin films [10]. Within the framework of this new treatment one may describe the dimensional $\mathrm{CQC}$ as a smooth change of the effective dimensionality $D_{\text {eff }}=(D+\delta)$ from $D$ to $(D+1)$ when the ratio $\left(\lambda_{T} / \xi\right)$ changes from zero to infinity.

The above scheme substitutes the integration (21) of the sum $S_{1}\left(t_{0}, k\right)$ by a double integral: a $D$-dimensional integral over the wave vector $\mathbf{k}$ and a $\delta(\leq 1)$-integration over the frequency $\nu$. The RG investigations within this scheme are quite clumsy and one may meet difficulties in higher orders of the loop expansion. The RG approach can be rationalized by substituting the double $(D, \delta)$ integration with a single $(D+\delta)$-dimensional integration. This change does not introduce any effect on the results [10].

Finally, we shall note that the same $R G$ results can be obtained by the formal replacement of the dynamical critical exponent $z=1$ in Eq. (46) by $\delta$.

\section{CONCLUDING REMARKS}

Although the MF properties of TIM are known in many respects, we have been able to obtain several new properties of the phase transitions along the HT and LT lines $A-J$ and $a-a$. The classical and quantum versions of the Ginzburg criterion have been derived and applied to TIM. The critical regions of the phase transitions have been determined. It has been shown that the critical regions along the HT and LT lines $A-J$ and $a-a$ are larger than the usual critical regions along the lines $0-J$ and $b-b$. Besides, it has been demonstrated that a critical region of classical (thermal) fluctuations of the order parameter at zero temperatures exists and is even larger than it might be expected.

Further, we have confirmed within both the MF approximation and the RG that there is a complete correspondence $(T \leftrightarrow \Gamma)$ between the HT and LT phase transitions only along the lines $0-J$ and $b-b$. However, the same correspondence $T \leftrightarrow \Gamma$ is valid only for these cases. The HT properties of the phase transitions along the lines $A-J$ and $a-a$ are quite different from those of the phase transitions along the LT lines $A-J$ and $a-a$. All these properties have been obtained in domains of the phase diagram $(T, \Gamma)$ where the quantum effects are irrelevant. Therefore, there exists a high-to-low temperature crossover (HLTC) of the critical properties of TIM. The HLTC does not produce any effective change of the spatial dimensionality but it exhibits a significant difference between the HT and LT critical properties along the lines $A-J$ and $a-a$.

The HLTC has been treated by the MF, GC and RG. The GC shows well established regions of validity of the classical (MF) transitions including large parts of the LT domain of the phase diagram. The RG reveals a conventional behaviour of a single classical phase transition, namely, the phase transition corresponding to the usual Ising model $(\Gamma=0)$. The other phase transitions in the classical HT and LT domains of the phase diagram exhibit quite unusual properties.

The CQC is a result of two reasons. One of them is the widely discussed quantum effects. The second reason, which has not been established up to now, consists in the linear LT dependence of the interaction parameter $u_{0}$ on the temperature. The linear dependence $u_{0} \sim T$ at LT has no quantum origin. A small deviation from the linearity $\left(u_{0} \sim T\right)$ could reflect in a break down of the $\mathrm{CQC}$ in its present form. For example, if $u_{0} \sim T^{\sigma}$ with $\sigma>1$, the CQC will be a crossover from the universality class $(1, D)$ at finite temperatures and $D>2$ to the universal MF behaviour at $T=0$. One may speculate that a more precise derivation of the field counterpart (2) of TIM may lead to a non-linear dependence $u_{0}(T)$. It should be emphasized that this can hardly happen. A simple dimensional analysis shows that the relation $u_{0} \sim T \sim \lambda_{T}$ should be valid irrespectively of the accuracy in the derivation of the $\phi^{4}$-model (2). 
Besides, the RG has been applied to demonstrate that the dimensional $\mathrm{CQC}$ due to the quantum correlations of the system occurs only for a very specific case, namely, for the phase transition at the zero-temperature critical point. The onset of an effective occurrence of the dimensional CQC can be observed in experiments at extremely low temperatures under the conditions determined in this paper.
[1] See, e.g., J. A. Hertz, Phys. Rev. B 14, 1165 (1976); G. Busiello and L. De Cesare, Phys. Lett. A 77, 177 (1980); D. I. Uzunov, Phys. Lett. A 87, 11 (1981); K. Lukierska-Walasek, Phys. Lett. A 95, 377 (1983); K. Lukierska-Walasek and K. Walasek, Phys. Lett. A 81, 527 (1981).

[2] See, e.g., D. I. Uzunov, Introduction to the Theory of Critical Phenomena (World Scientific, Singapore, 1993).

[3] See, e.g., A. M. J. Schakel, Nucl. Phys. B 453, 705 (1995); A. Caramico D'Auria, L. De Cesare and I. Rabuffo, Physica A 225, 363 (1996); T. R. Kirkpatrick and D. Belitz, Phys. Rev. Lett. 76, 2571 (1996); A. Caramico D'Auria, L. De Cesare, U. Esposito, and I. Rabuffo, Physica A 243, 152 (1997); A. M. J. Schakel, Phys. Lett. A 224, 287 (1997); S. L. Sondhi, S. M. Girvin, J. P. Carini, and D. Shahar, Rev. Mod. Phys. 69, 315 (1997); A. M. J. Schakel, Boulevard of Broken Symmetries, a preprint of Freie University of Berlin (1997).
[4] R. B. Stinchcombe, J. Phys. C 6, 2459 (1973).

[5] P. G. De Gennes, Solid State Commun. 1, 132 (1963); R. Brout, K. A. Müller and H. Thomas, Solid State Commun. 4, 507 (1966); M. Tokunaga and T. Matsubara, Progr. Theor. Phys. 35, 581 (1966).

[6] R. J. Elliott, P. Pfeuty and C. Wood, Phys. Rev. Lett. 25, 443 (1970); P. Pfeuty and R. J. Elliott, J. Phys. C 4, $2370(1971)$.

[7] A. P. Young, J. Phys. C 8, L309 (1975).

[8] I. D. Lawrie, J. Phys. C 11, 1123 (1978).

[9] See, e.g., D. I. Uzunov, in Lectures on Cooperative Phenomena in Condensed Matter, edited by D. I. Uzunov (Heron Press, Sofia, 1996), p. 46.

[10] L. De Cesare, L. Craco, I. Rabuffo, and D. I. Uzunov, J. Phys. Stud. 2, 175 (1998); L. De Casare, L. Craco, I. Rabuffo, I. P. Takov, and D. I. Uzunov. (1998) (unpublished). 